\title{
Coronal Condensation in Funnel Prominences as Return Flows of the Chromosphere-Corona Mass Cycle
}

\author{
Wei Liu ${ }^{1}$, Thomas E. Berger ${ }^{2}$ and B. C. Low ${ }^{3}$ \\ ${ }^{1}$ Stanford-Lockheed Institute for Space Research, HEPL Solar Physics, 452 Lomita Mall, \\ Stanford, CA 94305-4085, USA; email: weiliu@sun.stanford.edu \\ ${ }^{2}$ National Solar Observatory, 950 N. Cherry Avenue, Tucson, AZ 85719, USA \\ ${ }^{3}$ High Altitude Observatory, P.O. Box 3000, Boulder, CO 80307, USA
}

\begin{abstract}
We present SDO/AIA observations of a potentially novel type of prominence, called "funnel prominence", that forms out of coronal condensation at magnetic dips.

They can drain a large amount of mass (up to $\sim 10^{15} \mathrm{~g}$ day $^{-1}$ ) and may play an important role as return flows of the chromosphere-corona mass cycle.
\end{abstract}

Keywords. Sun: chromosphere, Sun: corona, Sun: filaments, prominences, Sun: magnetic fields

It has recently been recognized that a significant portion of the mass of the solar atmosphere is cycled between the hot, tenuous corona and the underlying cool, dense chromosphere (Berger et al. 2011; McIntosh et al. 2012; see a review by T. Berger in this proceedings). Like the water cycle on Earth, hot plasma is transported upward into the corona in such forms as spicules and flux emergence manifested as prominence bubbles and plumes, while cool plasma condenses out of the corona and drains back to the chromosphere via vertical prominence threads and transient coronal rain. One likely cooling mechanism is the radiative cooling instability (e.g., Karpen \& Antiochos 2008) in magnetized plasma that effectively inhibits across-field thermal conduction.

Recent $S D O /$ AIA observations have revealed a potentially new type of prominence, called "funnel prominence", that is an evident manifestation of this cooling process. As shown in Fig. 1, its distinct feature is a funnel shape formed by the combination of dipped coronal loops best seen at $171 \AA$ and cool $304 \AA$ material appearing and draining from the dips. Usually an emission cloud lasting for hours progressively appears at lower heights and in cooler EUV channels from $211 \AA(\sim 2.0 \mathrm{MK})$ to $193 \AA(\sim 1.6 \mathrm{MK})$, and then $171 \AA(\sim 0.8 \mathrm{MK})$, indicating a continual cooling process that eventually leads to the in-situ condensation of the $304 \AA(\sim 0.08 \mathrm{MK})$ prominence material (Liu et al. 2012; Berger et al. 2012). Field extrapolation from SDO/HMI magnetograms confirmed the existence of such magnetic dips at the initial condensation site (Liu et al. in prep.), which provide access to a large coronal volume for cooling mass to be collected and channeled to its lower portion for condensation. The condensed mass subsequently drains along meandering paths down to the chromosphere, suggestive of cross-field slippage of cool, poorly ionized mass that can involve magnetic reconnection (Low et al. 2012a,b). We found an average drainage velocity of $30 \mathrm{kmps}$ at a slower than free-fall acceleration of g/ 6 (Liu et al. 2012). A moderate-sized funnel prominence can drain a significant mass $\sim 10^{15}$ g day $^{-1}$, comparable to a fraction of the entire corona or a typical CME mass, suggestive of its important role as return flows of the chromosphere-corona mass cycle.

In a preliminary survey of the first three years (2010-2013, near the solar maximum) of $S D O /$ AIA data, we have found 13 funnel prominences as summarized in Table 1 . They appear off-limb at various locations predominantly at high latitudes $>50^{\circ}$, consisting of a single or multiple columns on the order of $70 \mathrm{Mm}$ tall and $20 \mathrm{Mm}$ wide 


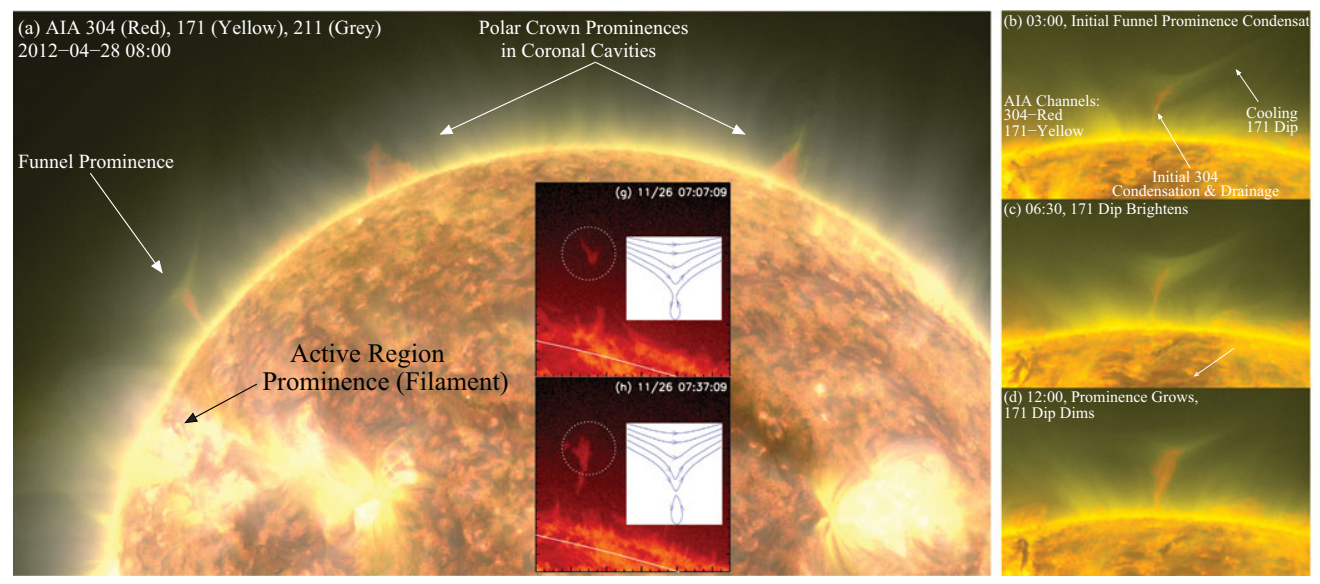

Figure 1. (a) Composite SDO/AIA $304 \AA$ (red), $171 \AA$ (yellow), and $211 \AA$ (grey) images showing a funnel prominence and two classical types of prominences. The inset, taken from Fig. 2 of Liu et al. (2012), shows initially a V-shaped $304 \AA$ condensation in another funnel prominence and a possible magnetic field configuration. (b)-(d) Enlarged view of the funnel prominence in (a) rotated to the local vertical up showing a cooling/condensation sequence indicated by the changing dip brightness and prominence size.

Table 1. Preliminary sample of 13 funnel prominences detected by SDO/AIA.

\begin{tabular}{|c|c|c|c|c|c|c|c|c|c|c|c|c|c|}
\hline \multirow{4}{*}{$\begin{array}{l}\text { Year } \\
\text { Date } \\
\text { Location }\end{array}$} & \multirow{3}{*}{$\begin{array}{l}2010 \\
11 / 26 \\
\text { W } 86 \mathrm{~S} 13\end{array}$} & \multirow{3}{*}{$\begin{array}{l}2011 \\
10 / 14 \\
\text { E } 100 \mathrm{~N} 61\end{array}$} & \multicolumn{8}{|l|}{2012} & \multicolumn{3}{|l|}{2013} \\
\hline & & & $03 / 15$ & $03 / 17$ & $04 / 28$ & $06 / 24$ & $06 / 27$ & $06 / 29$ & $07 / 13$ & $09 / 30$ & $05 / 11$ & $06 / 21$ & $07 / 13$ \\
\hline & & & W 79 N 57 & $\mathrm{~W} 40 \mathrm{~N} 80$ & E $83 N 39$ & W $88 \mathrm{~S} 42$ & E $91 \mathrm{~N} 58$ & E $92 \mathrm{~N} 68$ & E $90 N 75$ & W $95 \mathrm{~N} 63$ & W 82 N 52 & E $87 N 77$ & E $96 \mathrm{~N} 68$ \\
\hline & No & No & No & Yes & No & No & No & No & No & Yes & $\mathrm{No}$ & Yes & No \\
\hline
\end{tabular}

(e.g., Fig. 1) with lifetime of hours to a day or two. Some funnel prominences disappear probably because of depletion by drainage, while others erupt together with their hosting magnetic dips. These characteristics distinguish funnel prominences from active region prominences and quiescent polar-crown prominences within coronal cavities situated along polarity inversion lines (PILs) (see Fig. 1); they may or may not require a PIL or flux-rope cavity. Meanwhile, funnel prominences share some morphological similarities with quiescent prominences, such as the conical shapes at the tops of funnels and the "horns" in quiescent prominences (Berger 2012), both seen as $171 \AA$ emission. Funnel prominences are also similar to "cloud prominences" (Lin et al. 2006) in that they both involve downflows streaming out of high-altitude condensations. However, some spidershaped cloud prominences show drainage along well-defined curved paths at close to free-fall speeds (Allen et al. 1998), resembling coronal rain sliding down coronal loops, while funnel prominences drain along meandering paths at much lower speeds (yet $2-3$ times faster than those of quiescent prominences) possibly due to cancellation of gravity by a Lorentz force at the magnetic dips. Further analysis is underway to validate funnel prominences as a new prominence type and to investigate their physical nature.

\section{References}

Allen, U. A., Bagenal, F., \& Hundhausen, A. J. 1998, in ASP-CS, Vol. 150, ed. D. F. Webb, et al. 290 Berger, T. 2012, in ASP-CS, Vol. 463, ed. T. R. Rimmele, A. Tritschler, F. Wöger, et al. 147

Berger, T. E., Testa, P., Hillier, A., et al. 2011, Nature, 472, 197

Berger, T. E., Liu, W., \& Low, B. C. 2012, ApJ, 758, L37

Karpen, J. T. \& Antiochos, S. K. 2008, ApJ, 676, 658

Lin, Y., Martin, S. F., \& Engvold, O. 2006, BAAS, 38, 219; AAS/SPD Meeting \#37, \#1.21

Liu, W., Berger, T. E., \& Low, B. C. 2012, ApJ, 745, L21

Low, B. C., Berger, T. E., Casini, R., \& Liu, W. 2012a, ApJ, 755, 34

Low, B. C., Liu, W., Berger, T. E., \& Casini, R. 2012b, ApJ, 757, 21

McIntosh, S. W., Tian, H., Sechler, M., \& De Pontieu, B. 2012, ApJ, 749, 60 